\title{
ERZEUGT DIE NATURWISSENSCHAFTLICHE ERKENNTNIS NUR WISSEN VOM ENDLICHEN, WÄHREND DAS WAHRHAFT UNENDLICHE NUR GEGLAUBT WERDEN KANN?
}

\section{Endliches und wahrhaft Unendliches}

In der Enzyklopädie kann man über die »schlechte« und »wahrhafte« Unendlichkeit folgendes lesen:

"Etwas wird ein Anderes, aber das Andere ist selbst ein Etwas, also wird es gleichfalls ein Anderes und so fort ins Unendliche. 1

"Diese Unendlichkeit ist die schlechte oder negative Unendlichkeit, indem sie nichts ist, als die Negation des Endlichen, welches aber ebenso wieder entsteht, somit ebensosehr nicht aufgehoben ist, - oder diese Unendlichkeit drückt nur das Sollen des Aufhebens des Endlichen aus. Der Progreß ins Unendliche bleibt bei dem Aussprechen des Widerspruchs stehen, den das Endliche enthält, daß es sowohl Etwas ist als sein Anderes, und ist das perennierende Fortsetzen des Wechsels dieser einander herbeiführenden Bestimmungen. ${ }^{2}$

»Was in der That vorhanden ist, ist, daß Etwas zu Anderem und das Andere überhaupt zu Anderem wird. Etwas ist im Verhältnis zu einem Anderen selbst schon ein Anderes gegen dasselbe; somit da das, in welches es übergeht, ganz dasselbe ist, was das, welches übergeht - beide haben keine weitere als eine und dieselbe Bestimmung, ein Anderes zu sein -, so geht hiermit Etwas in seinem Übergehen in Anderes nur mit sich selbst zusammen, und diese Beziehung im Übergehen und im Anderen auf sich selbst ist die wahrhafte Unendlichkeit. Oder negativ betrachtet: was verändert wird, ist das Andere, es wird das Andre des Anderen. So ist das Sein, aber als Negation der Negation, wieder hergestellt und ist das Fürsichsein. $\aleph^{3}$

Gemäß Hegel beschränkt sich der wissenschaftliche Empirismus (worunter er auch die Naturwissenschaften subsumiert) dem Inhalte nach auf Endliches (bzw. er weiß nur von der schlechten Unendlichkeit). Dieser Denkart sei es geschuldet, daß er das wahrhaft Unendliche bzw. das Übersinnliche überhaupt als Erkennbares leugnet: „Die konsequente Durchführung des Empirismus, insofern er dem Inhalte nach sich auf Endliches beschränkt, leugnet aber das Übersinnliche überhaupt oder wenigstens die Erkenntnis und Bestimmtheit desselben und läßt dem Denken nur die Abstraktion und die formelle Allgemeinheit und Identität zu. ${ }^{4}$

Ähnliche Ausführungen findet man in der Logik. Und schon in Hegels Frühschrift Glauben und Wissen findet man die folgende Überlegung: Nach dem festen Prinzip dieses Systems der Bildung (womit Hegel den nur auf die Glückseligkeit des Subjekts ausgerichteten Eudämonismus und die Aufklärung, d. h. den Empirismus und die Philosophie Kants, Jacobis und Fichtes meint) ist das Endliche an und für sich und absolut, es ist die einzige Realität. Auf der einen Seite steht damit das Endliche und Einzelne selbst in der Form der Mannigfaltigkeit, und in diese wird alles Religiöse, Sittliche und Schöne geworfen, weil es fähig ist, vom Verstande als ein Einzelnes begriffen zu werden. Auf der anderen Seite steht die absolute Endlichkeit in der Form des Unendlichen als Begriff. Das Unendliche und Endliche, die nicht in der Idee identisch gesetzt werden sollen, denn jedes ist absolut für sich, stehen auf diese Weise in der Beziehung des Beherrschens gegeneinander, denn im absoluten Gegensatz derselben ist der Begriff das Bestimmende. Über diesem absoluten Gegensatz und den relativen Identitäten des Beherrschens und der empirischen Begrifflichkeit steht das Ewige, das Nichtzuberechnende, Unbegreifliche, Leere, ein unerkennbarer Gott. ${ }^{5}$

In Hinblick auf die in der Überschrift formulierte Frage lautet Hegels Antwort also: Ja, die Naturwissenschaften haben nur Wissen vom Endlichen, denn ihr Wissen ist Verstandeswissen. Nur die Vernunft, 
mithin die Philosophie, hat Wissen vom wahrhaft Unendlichen und kann das Unendliche mit dem Endlichen vermitteln.

\section{Mathematisch Unendliches und wahrhaft Unendliches}

Nun scheint dieser Bestimmung der Fachwissenschaften aber Hegels Begeisterung für den Begriff des mit dem Infinitesimalkalkül eingeführten mathematischen Unendlichen entgegenzustehen. Denn dieser Kalkül war für die und zusammen mit der Newtonschen Bewegungslehre entwickelt worden, und, indem er integraler Teil dieser Lehre war, war damit auch das mathematisch Unendliche tragend für diese Lehre. Man könnte daher erwarten, daß Hegels Begeisterung für das mathematisch Unendliche auch seine Sicht auf die Mechanik, also auf die erste physikalische Dynamik, verändert hätte. Im folgenden soll dargelegt werden, daß $\mathrm{He}$ gel das mathematisch Unendliche aber so rezipiert, daß es zu keinem Wandel seiner Sicht auf die Naturwissenschaften kommt.

In seinen expliziten Ausführungen zum Infinitesimalkalkül, die hauptsächlich in den Anmerkungen zum Abschnitt „Die Unendlichkeit des Quantums« enthalten sind, zeigt Hegel, daß das mathematische Unendliche dieses Kalküls dem Begriff des wahrhaft Unendlichen entspringt, »indem das Quantum, indem es unendlich ist, als ein aufgehobenes, als ein solches zu denken gefordert wird, das nicht ein Quantum ist und dessen quantitative Bestimmtheit doch bleibt «. ${ }^{6}$ Hegel akzeptiert auch das Lagrangesche Verfahren, mathematische Funktionen der Mechanik in eine Reihe zu entwickeln und Glieder der Reihe nicht nur wegen ihrer relativen Kleinheit, sondern auch aus physikalischen Gründen zu vernachlässigen. Für ihn ist das ein annehmbares Vorgehen, da die Glieder der Reihe hiermit »nicht nur als Teile einer Summe», sondern »als qualitative Momente eines Ganzen des Begriffs « angesehen werden. »Hierdurch« - schreibt Hegel weiter "erhält das Weglassen der übrigen Glieder, die der schlecht unendlichen Reihe angehören, eine gänzlich verschiedene Bedeutung von dem Weglassen aus dem Grunde der relativen Kleinheit derselben. « 7

Das, was Hegel dem Mathematiker und Physiker Lagrange zugesteht, ist allerdings nicht akzeptabel für den Philosophen Hegel. Das begriffslogische Anliegen, das Hegel motiviert, den Infinitesimalkalkül so überaus ausführlich zu diskutieren, liegt im Nachweis des qualitativen Charakters der dabei in Rede stehenden Größenform. Diesen Charakter sieht Hegel »am unmittelbarsten in der Kategorie der Grenze des Verhältnisses « gefaßt. ${ }^{8}$ Er diskutiert daher den Begriff der Grenze und polemisiert lebhaft gegen ihr Verständnis als etwas, das nur durch unendliche Annäherung erreicht wird, also gegen ihr Verständnis als schlechte Unendlichkeit. Ihm zufolge liegt in der Vorstellung der Grenze die von der Begriffslogik entwickelte wahrhafte Kategorie der qualitativen Verhältnisbestimmung der veränderlichen Größen. Denn $d x$ und $d y$ als Formen dieser veränderlichen Größen werden nur als Momente eines Ganzen angesehen, nämlich von $d x / d y$, das seinerseits als ein Unteilbares genommen wird. ${ }^{9}$

Hegel kritisiert also die durch die Mathematik gegebene Begründung des Differentialkalküls als philosophisch unzulänglich, mehr noch, er meint, die Anwendung des Kalküls auf die Mechanik, d.h. auf die Bewegung, verwische den reinen Gedanken dieses Kalküls. Akzeptiert man diesen Standpunkt ohne Einschränkung, so bleibt man im Hegelschen Gedankenkosmos. Die Mathematik hat dann mit dem mathematisch Unendlichen etwas gewonnen, das dem wahrhaft Unendlichen entspringt, kann aber dessen Rechtfertigung nur mit Bezug auf die Anschauung liefern, während die wahre Begründung, die Begründung im wahrhaft Unendlichen, nur die Philosophie geben kann.

Kann man aber diesen Standpunkt in Ansehung der zu Hegels Zeiten (und erst recht heute) vorliegenden Naturwissenschaften akzeptieren? Man wird Hegel ohne weiteres folgen können, wenn er die bis zu seiner Zeit gegebene Begründung des Differentialkalküls kritisiert, insbesondere kann man ihm darin zustimmen, daß die Mathematik dabei auf die Erfahrung rekurrierte. Man kann sogar sagen, die Mathematik muß auf sie Bezug nehmen, will sie nicht im rein Abstrakten bleiben. Sie kann das Qualitative nur aus der Erfahrung nehmen, nicht aus sich selbst heraus entwickeln. Es bedarf mithin - will man die durch die Infinitesimalrechnung eröffneten Möglichkeiten dennoch realisieren - eines Konzepts physikalischer Erfahrung, eines Konzepts, das physikalische Erfahrung nicht mit Alltagserfahrung identifiziert, eines Konzepts, das die Spezifik wissenschaftlicher Erfahrung erkennt. 
Anderes gilt für die Philosophie. Dem philosophischen Begriff - auch darin kann man Hegel folgen ist die Fähigkeit, das Qualitative zu entwickeln, durchaus zuzugestehen. Jedoch übersah Hegel, daß der philosophische Begriff diese Fähigkeit nur unter der Voraussetzung des sogenannten Prinzips wissenschaftlicher Erfahrung haben kann, was die Fähigkeit des philosophischen Begriffs nicht unwesentlich variiert. 10

So erkennt Hegel zwar als einer der ersten die große Bedeutung dieses Kalküls. Er übersieht aber einen Teil des eigentlichen Clous desselben, nämlich seine Fähigkeit, die physikalische Bewegungsfassung zu ermöglichen. Er übersieht dies, weil er das mathematisch Unendliche durch die Brille seines System rezipiert. An die Stelle des Begriffs der Funktion und ihrer Ableitungen tritt der begriffslogische Quotient. Der Unterschied zwischen Hegels begriffslogischem Quotienten und einem echten Funktionsbegriff besteht nicht nur darin, daß letzterer eine mehr als zweistellige Struktur beinhaltet, sondern auch im Wechselspiel zwischen festgelegten bzw. frei gewählten und folgenden Größen, dem Denken von bestimmten Änderungen (die nicht auf das Negative oder das Andere zu reduzieren sind) und der Darstellung des Zusammenhanges zwischen den Änderungen - sowohl zwischen den Änderungen gleichen Grades, aber verschiedener Qualitäten, wie auch zwischen den Änderungen verschiedenen Grades. ${ }^{11}$

Durch diese Verdrillung wird der Differentialkalkiil von der physikalischen Bewegungsfassung abgehoben und zu einer Ahnung der Mathematik vom wahrhaft Unendlichen verwandelt und so zu einem in das Hegelsche System Einfügbaren gemacht. Und so bleibt für Hegel dann auch die Stellung der Naturwissenschaft zum Unendlichen vom Infinitesimalkalkül unbeeinträchtigt, und er kann die Naturwissenschaft weiter als Empirismus, als reines Verstandeswissen charakterisieren.

Die von Hegel vorgenommene Isolierung des Infinitesimalkalüls von seiner physikalischen Begrifflichkeit verspertt ihm insbesondere die Einsicht darein, daß die Naturwissenschaft in Form der Newtonschen Dynamik auf dem vom ihm philosophisch reflektierten neuzeitlichen Denkprinzip basiert, das eine Neubestimmung der Beziehung von Gegenstand und Verhältnis vornimmt - derart, daß das Verhalten selbst zum Gegenstand werden kann, und daß der Charakter der Infinitesimalrechnung nur auf der Grundlage dieses Prinzips verstanden werden kann. Oder anders gesagt: Der Realitätscharakter der Infinitesimalrechnung, mithin der untrennbare Zusammenhang dieses mathematischen Kalküls mit dem physikalischen Bewegungsbegriff oder der physikalischen Fassung der Bewegung, kann nicht begriffen werden, ohne den Unterschied zwischen dem antiken und dem neuzeitlichen Denkprinzip zu begreifen. ${ }^{12}$ Die Suche nach dem Gesetz ist - seit dem à $\rho x \eta^{\prime}-$ Gedanken - die Suche nach dem Bleibenden im Wechsel der Erscheinungen. Das Bleibende ist das, was ist, das Sein. Wird dieses als Substanz im antiken Sinne gefaßt, gelangt die Wissenschaft nur bis zur (Lage-)Geometrie, kinematischen Astronomie und zur Statik, verstanden als praktische

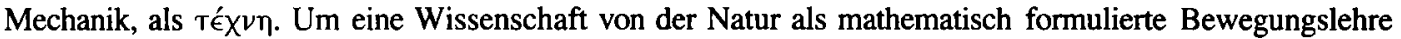
(Dynamik) zu begrïnden, muß das Sein, das Bleibende im Wechsel, als veränderlich, als in sich bewegt gedacht werden können. Durch das Denkprinzip, das Lasswitz das »Denkmittel der Variabilität« nennt, ${ }^{13}$ konnte die Kontinuität in der erforderlichen Weise gefaßt werden. Denn die Realität der Dinge, das Sein, ist nicht garantiert durch die Substantialität, da dies eine Relation ist, die zwischen schon Gegebenem vermittelt. Es mußte ein Prinzip gefunden werden, das das Sein so faßt, daß es den Begriff der Veränderlichkeit in sich einschließt, ein Prinzip, das es gestattet, das »Ding «, die Einheit, so zu denken, daß sie in sich Ausgangspunkt einer gesetzlichen Entwicklung ist. ${ }^{14}$

Die Infinitesimalrechnung ist das Mittel, das geeignet ist, dieses Prinzip (mathematisch) zu realisieren. Zugleich kann sie philosophisch adäquat nur auf der Grundlage des neuzeitlichen Prinzips gedacht werden. Der Übergang vom antiken zum neuzeitlichen Denkprinzip realisiert sich mathematisch im Übergang von der Methode der Indivisiblen zur Infinitesimalrechnung, von der Addition zum Fließen.

\section{Fazit: Die Naturwissenschaft überschreitet die Grenze des Endlichen}

Um es zu wiederholen, es ist also so, daß die Bewegung, das Verhalten als Gegenstand, als Gegenstand der Wissenschaft nur erkannt werden kann, wenn das Sein als »Funktion«, als ein bestimmt strukturiertes Werden gedacht wird. Das Unendliche, und zwar das im Hegelschen Sinne mit dem Endlichen eine Einheit bildende Unendliche, wird in der Funktion realisiert. Dabei ist die mathematische Fassung natürlich noch kein philosophischer Begriff, sondern eine Festschreibung für einen bestimmten Zweck. Doch ohne die gründli- 
che, und zwar immer wieder neue, philosophische Analyse der fachwissenschaftlichen Ergebnisse und Begriffe gibt es letztlich keinen philosophischen ProgreB.

Indem Hegel den Zusammenhang zwischen dem Infinitesimalkalkül und der physikalischen Bewegungsfassung nicht erkennt, bleiben ihm Einsichten in wesentliche Aspekte dieses Kalküls und des Status der Naturwissenschaften verschlossen. Zwar sind die Naturwissenschaften insofem endliche Wissenschaften, als sie nicht das Unendliche der Philosophie im Sinne des Totalen, des Absoluten zum Gegenstand haben. Das mathematische Unendliche des Infinitesimalkalküls steht aber dem wahrhaft Unendlichen näher, als es Hegel ihm zugibt, denn es ermöglicht die bzw. realisiert sich in der Bewegungsfassung.

Mittels der Infinitesimalrechnung und somit des mit ihr eingeführten Begriffs des Unendlichen kann die Bewegung gefaßt werden, und zwar nicht etwa als bloße Aufeinanderfolge punktueller Ereignisse und Ruhelagen, sondern durchaus im Sinne der Bestimmung von Hegel als Widerspruch - natürlich in einer für die mathematisch-physikalische Betrachtungsweise typischen Form. ${ }^{15}$ Ohne in logische Widersprüche zu geraten, gelingt es, den Sachverhalt, daß ein bewegter Körper zu ein und demselben Zeitpunkt an einem Ort ist und nicht an ihm ist, darzustellen (in einer Weise, die die Berechnung und Messung der Bewegung möglich macht). Dieses Unendliche des Infinitesimalkalküls, und zwar das im Hegelschen Sinne mit dem Endlichen eine Einheit bildende Unendliche, ist es, welches es möglich macht, den daseienden Widerspruch, der nach Hegel die Bewegung ausmacht, zu fassen. Damit überschreitet die Naturwissenschaft die Grenze des Endlichen und auch die der schlechten Unendlichkeit, damit ist sie nicht nur reines Verstandeswissen.

Prof. Dr. Horst-Heino v. Borzeszkowski

Technische Universität Berlin

Institut für Theoretische Physik

Hardenbergstr. 36

10623 Berlin

borzeszk@itp.physik.tu-berlin.de

\section{ANMERKUNGEN}

1 G.W.F. HEGEL, Enzyklopädie der philosophischen Wissenschaften im Grundrisse, Erster Teil, in: Werke in $20 \mathrm{Bdn}$., auf der Grundlage der Werke von 1832-1845 neu edierte Ausgabe, Redaktion E. Moldenhauer und K.M. Michel, Frankfurt/M. 1986, Bd. 8, 198 (§93).

2 Ebd., $199(\$ 94)$.

3 Ebd., 200 f. $(\$ 95)$.

4 Ebd., $108(\S 38 \mathrm{~A})$.

5 Vgl. G.W.F. HEGEL, "Glauben und Wissen oder Reflexionsphilosophie der Subjektivităt in der Vollstăndigkeit ihrer Formen als Kantische, Jacobische und Fichtesche Philosophie«, in: Werke, Bd. 2, 293.

6 G.W.F. HEGEL, Wissenschaft der Logik. Erster Teil, in: Werke, Bd. 5, 283.

7 Ebd., 309.

8 Vgl. ebd., 312, siehe auch 298, 318-320.

9 Vgl. ebd., 312.

10 Ausführlicher zu dieser Problematik siehe H.-H. v. BORZESZKOWSKI und R. WAHSNER, Physikalischer Dualismus und dialektischer Widerspruch. Studien zum physikalischen Bewegungsbegriff, Darmstadt 1989; dies., Die Wirklichkeit der Physik. Studien zu Idealität und Realität in einer messenden Wissenschaft, Frankfurt/M. u. a. 1992, 239-285.

11 Ausführlicher zu dieser Problematik siehe: R. WAHSNER, "mer Gedanke kann nicht richtiger bestimmt werden, als Newton ihn gegeben hat.، Das mathematisch Unendliche und der Newtonsche Bewegungsbegriff im Lichte des begriffslogischen Zusammenhangs von Quantität und Qualität«, in: Hegels Seinslogik - Interpretationen und Perspektiven, hg. von A. Amdt und Ch. Iber, Berlin 2000; H.-H. v. BORZESZKOWSKI und R. WAHSNER, "Infinitesimalkalkül und neuzeitlicher Bewegungsbegriff oder Prozeß als Größe«, in: Jahrbuch für Hegelforschung 2002, Sankt Augustin (im Druck), auch: Preprint 165 des MPI für Wissenschaftsgeschichte, Berlin 2001.

12 Zu diesem Umbruch vgl: K. LASSWITZ, Geschichte der Atomistik, Hamburg/Berlin 1890; DERS., "Zum Problem der Continuität«, in: Philosophische Monatshefte, 24 (1888), 9-36; E. CASSIRER, Substanzbegriff und Funktionsbegriff. Untersuchungen über die Grundlagen der Erkenntniskritik, Darmstadt 1990; DERS., Das Erkenntnisproblem in der Philosophie und Wissenschaft der neueren Zeit, Darmstadt 1994, Bd. I-IV, insbes. Bd. I, 18-61; DERS., Die Philosophie der Aufklärung, Tübingen 1932, 1-122. Siehe 
auch: E.J. DIJKSTERHUIS, Die Mechanisierung des Weltbilds, Berlin/Göttingen/Heidelberg 1956; M. WOLFF, Geschichte der Impetustheorie. Untersuchungen zum Ursprung der klassischen Mechanik, Frankfurt/M. 1978; B. HEIDTMANN, "Die sich selbst bewegende Substanz. Zu Voraussetzungen und Konsequenzen des philosophischen Grundsatzprogramms Hegels«, in: Arbeit und Reflexion. Zur materialistischen Theorie der Dialektik - Perspektiven der Hegelschen Logik, hg. von P. Furth, Köln 1980; R. WAHSNER, Mensch und Kosmos. Die copernicanische Wende, Berlin 1978, insbes. 6-70, 95-359; DIES., Zur Kritik der Hegelschen Naturphilosophie. Über ihren Sinn im Lichte der heutigen Naturerkenntnis, Hegeliana. Studien und Quellen zu Hegel und zum Hegelianismus, hg. von H. Schneider, Bd.7, Frankfurt/M. u. a. 1996, 11-19, 54-60, 116-121, 217-221; H.-H. v. BORZESZKOWSKI und R.

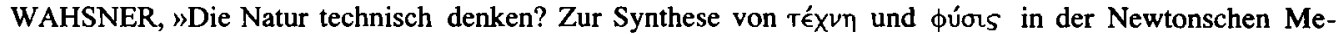
chanik oder das Verhältnis von praktischer und theoretischer Mechanik in Newtons Physik «, in: Preprint 87 des MPI für Wissenschaftsgeschichte, Berlin 1998, insbes. 9-12, 19-26.

13 Vgl. LASSWITZ, "Zum Problem der Continuität«, 17.

14 Vgl. ebd., 18.

15 Siehe v. BORZESZKOWSKI und WAHSNER, Physikalischer Dualismus und dialektischer Widerspruch. 